\title{
Consumer Choice Strategies for Comparing Noncomparable Alternatives*
}

\author{
MICHAEL D. JOHNSON**
}

\begin{abstract}
Research on consumer choice has focused on easily comparable alternatives, a subset of the choices consumers regularly face. This paper outlines the problem and two general strategies for comparing noncomparable alternatives, a subset of choices that has been overlooked in the literature. Experiments are reported that support use of the strategies.
\end{abstract}

$\mathrm{C}$ onsumers choosing among brands in the same product category face alternatives that are generally described or represented by the same attributes. This allows consumers to directly compare the alternatives. Consumers compare televisions, for example, on screen size and picture quality-or soft drinks on sweetness and flavor. Situations involving such comparable choice alternatives may arise as the direct result of a hierarchical choice process (Bettman 1974; Howard 1977), where consumers choose among successively more concrete or specific categories or groups of alternatives. The only specific alternatives or brands compared tend to be quite similar.

Some situations require a choice among specific alternatives from different product categories, often called generic competition (Kotler 1984). Immediate environmental constraints may, for example, create situations where only one alternative is available in each category. Even when more than one alternative is available in each category, consumers may first choose the best alternative in each category and then compare the specific category choices. Consider choosing between a television and a refrigerator, or a

\footnotetext{
* This article was submitted, edited, and typeset via diskette.

** Michael D. Johnson is Assistant Professor of Marketing at the University of Michigan's Graduate School of Business Administration, Ann Arbor, MI 48109. The author thanks Hillel Einhorn, Harry Davis, Michael Ryan, Robin Hogarth, Robert Libby, Thomas Nagle, Robert Blattberg, and three anonymous reviewers for valuable comments, discussions, and insights on this topic. Special thanks to J. Edward Russo for his enduring involvement in this research and his guidance over the years. The assistance of Jill Johnson, Michael Crawford, and Dudley Luke in compiling and coding the experimental data is gratefully acknowledged. Financial assistance was provided by the Red and White Foundation and by the Consumer Behavior Laboratory at the University of Chicago's Graduate School of Business, which also provided laboratory facilities.
}

television and a Hawaiian vacation. Since each of these alternatives is naturally described by a different set of attributes, immediate and direct comparisons may be difficult. How can one compare a television's screen size with a refrigerator's freezer space or a vacation's location? When alternatives are described by different attributes, they are relatively "noncomparable," yet comparisons are made on some basis.

Consumer choice is not limited to comparable alternatives. Rather, choice alternatives vary in comparability, where comparability is the degree to which alternatives are described or represented by the same attributes. However, studies investigating the details of how consumers go about making decisions-or "process tracing" studies-have focused on comparable alternatives (Berning and Jacoby 1974; Bettman and Jacoby 1976; Bettman and Kakkar 1977; Bettman and Park 1980; Jacoby, Chestnut, Weigl, and Fisher 1976; Jacoby, Szybillo, and Busato-Schach 1977; Lussier and Olshavsky 1979; Payne 1976; Russo and Dosher 1983; Russo and Rosen 1975; Sheluga, Jaccard, and Jacoby 1979). Choices have been limited to alternatives within product categories even in studies involving more than one product category. In all but one study (Smead, Wilcox, and Wilkes 1981), alternatives overlapped completely on descriptive attributes.

The central problem of this research is to ascertain how comparability of alternatives affects decision processes. Of special interest is the as yet unstudied problem of how consumers compare noncomparable alternatives.

By focusing on our ability to compare values on the same attributes, comparability may not adequately describe some choice alternatives. Comparability naturally describes alternatives whose attributes are represented dimensionally. If, alternatively, product representations are more feature-based, the concept of comparable and noncomparable alternatives is more 
ambiguous. Comparability may be replaced by the more general notion of similarity in such cases, where similarity is a function of the number of common and distinctive features associated with the alternatives (Tversky 1977). The hypotheses that follow do, however, apply to both dimensional and feature-based representations.

\section{CHOICE STRATEGIES FOR COMPARABLE ALTERNATIVES}

To address how consumers compare noncomparable alternatives, consider first the goals motivating strategy selection and how, in turn, comparable alternatives are compared. At least two goals influence strategy selection. While striving to choose the best possible alternative, consumers put forth as little effort as possible. In other words, consumers try to minimize both error and effort when selecting a strategy (Hogarth 1975; Johnson 1980; Shugan 1980; Russo 1981; Wright 1975). Because error and effort reduction are often incompatible goals, consumers trade off error for effort.

Two classes of strategies exist for choosing among comparable alternatives. Strategies comparing alternatives directly on attributes - the so-called withinattribute strategies-include the additive difference rule (Tversky 1969) and elimination by aspects (Tversky 1972). They contrast with across-attribute strategies, such as the additive utility, conjunctive, and disjunctive strategies (Einhorn 1970). Across-attribute strategies evaluate alternatives holistically; that is, values across attributes are combined, and comparisons are based on resulting overall evaluations.

Because a comparison of values on the same attribute is often easier than combining values across attributes, within-attribute strategies are often preferred (Russo and Dosher 1983; Tversky 1969). Many process-tracing studies support the use of within-attribute strategies on comparable alternatives (Bettman and Jacoby 1976; Lussier and Olshavsky 1979; Russo and Dosher 1983; Russo and Rosen 1975; Smead, Wilcox, and Wilkes 1981). Of course, within-attribute strategies are not always relatively easy. A preference for withinattribute strategies, on the basis of minimizing effort, assumes that the format of attribute information is irrelevant. Information format is the location and organization of attribute information in the consumer's environment. If, for example, all information is centrally located, within-attribute comparisons are facilitated. In fact, format affects both the strategy chosenwithin-attribute versus across-attribute-(Bettman and Kakkar 1977) and the amount of information used (Russo 1977).

One must distinguish, however, between external representations, like a Consumer Reports matrix or an information board (Jacoby et al. 1976), and internal representations residing in short-term memory or consciousness. Many of the decisions outlined here involve internal representations. Environmental factors influencing external format are largely irrelevant. Because format is not important to the range of decisions under study, and because its effects are relatively well documented (Russo 1981), it is not studied here.

Still other factors on both sides of the error/effort tradeoff affect strategy selection. An experiment reported by van Raaij (1977), for example, shows that preference for within-attribute strategies decreases as attribute values become more extreme. Looking more at error, Klein (1983) shows a relationship between utility differences across attribute value levels and strategy use. The discussion here focuses specifically on the ease of within-attribute comparisons relative to across-attribute combinations and their role in strategy selection, even when alternatives are noncomparable. More complete formulations of strategy error and effort are developed elsewhere for both comparable (Johnson and Payne 1984) and noncomparable (Johnson 1984) choice alternatives.

\section{CHOICE STRATEGIES FOR NONCOMPARABLE ALTERNATIVES}

While within-attribute strategies require comparability among products, across-attribute strategies can be used directly on either comparable or noncomparable alternatives. As consumers simply combine attribute values into overall evaluations on which a direct comparison is made, attribute comparisons are unnecessary. For example, using a linear compensatory strategy, a television's screen size, picture quality, and price, or a stereo's sound quality, power, and price are combined into an overall evaluation of "value." The consumer then compares the alternatives on overall net value. As overall evaluations are themselves quite abstract, they form a common basis of comparison. This, of course, is how economists deal with comparability. Considering alternatives on overall value or "utility" makes all alternatives comparableeven guns and butter.

Overall evaluations are not the only means of comparing noncomparable alternatives. Preference for direct, within-attribute comparisons on comparable alternatives suggests that consumers seek out comparable representations for noncomparable alternatives allowing within-attribute comparisons. For example, one can compare a stereo and a television on the basis of how much one uses or enjoys each alternative. A stereo and a refrigerator might be compared on the basis of necessity or practicality. Consumers may 
describe alternatives on whatever attributes they can be compared on and retain a within-attribute strategy.

To conceptualize the within-attribute strategy, think of attributes as lying along a continuum from the concrete to the abstract. ${ }^{1}$ When faced with initial concrete, noncomparable representations, the consumer will look for comparable attributes by representing alternatives at higher levels of abstraction. As attributes become more abstract, they also become common to more and more alternatives. ${ }^{2}$ Once a level of abstraction is reached where alternatives are comparable, a within-attribute strategy is possible. Price is, of course, one concrete attribute on which even noncomparable alternatives can be compared, and is treated separately.

Consider, for example, three different alternatives: a refrigerator, a television, and a stereo. All three are described by different concrete attributes, making direct comparisons on descriptive nonprice attributes impossible. At more abstract levels, representations become increasingly comparable. At an intermediate level of abstraction, a stereo and a television may be represented and compared directly on entertainment value and social status. For example, "a television is more entertaining than a stereo, though a stereo confers more status." At a more abstract level, all three alternatives may be compared on necessity. For example, "a refrigerator is more of a necessity than either a stereo or a television." Alternatives that are noncomparable at a given level are made comparable by moving to a higher level of abstraction.

\section{Strategy Differences and Tradeoffs}

What the within-attribute strategy adds is the possibility of product comparisons at different levels of abstraction. The more noncomparable the alternatives, the higher one must go to make a direct comparison on attributes. In contrast, the across-attribute strategy involves a comparison at a single very abstract level corresponding to an overall evaluation. While the two strategies may appear similar (as when the withinattribute strategy is performed at an extreme level of abstraction), they are nonetheless different. The most abstract level in the within-attribute strategy may not be as abstract as an overall evaluation. Overall evaluations represent an ultimate level of abstraction.

\footnotetext{
${ }^{1}$ While various definitions of concreteness-abstractness exist in the psychological literature (cf. Paivio 1971), abstractness is typically the inverse of how directly an attribute denotes particular objects or events. It is equated with specificity-generality of terms and subordination-superordination of categories. This definition is adopted here, though abstractness-concreteness and generality-specificity may not be equivalent in all contexts.

${ }^{2}$ The continuum of attributes I propose is similar, though not identical, to Howard's (1977) evaluative hierarchy for generating choice criteria. He does not address actual product representations, comparability, or specific choice processes.
}

Also, while across-attribute processing may itself be considered an abstraction process, abstraction in the within-attribute strategy is qualitatively different from combining values across attributes.

Abstracting product representations in the withinattribute strategy is a two-stage process. To move from a concrete to an abstract representation, consumers must first construct a set of attributes that apply to the alternatives at the desired level of abstraction. Once constructed, consumers form values on the attributes for each alternative. For example, consider a choice between a motorcycle and an automobile. To represent these alternatives jointly on a more abstract level consumers might realize that both can be described in terms of "safety," "transportability," and "handling." Both the motorcycle and the automobile can then be evaluated on these attributes. This second stage may involve simply recalling values from memory or constructing them from existing concrete values.

Which strategy do consumers prefer for noncomparable alternatives? An advantage of the withinattribute strategy is that relatively easy within-attribute comparisons are retained; however, the within-attribute strategy requires another stage of processing-abstraction. An advantage of the across-attribute strategy is that it can be used directly without the abstraction stage necessary in the within-attribute strategy. Assuming that consumers minimize effort, this should result in increased use of the across-attribute strategy from comparable to noncomparable alternatives. At some point it should be easier to combine values across attributes into an overall evaluation than to raise individual attributes to a level of abstraction required for direct, within-attribute comparisons and compare the alternatives. Thus, while the withinattribute strategy may be retained by using more abstract attributes, the effort required by the added abstraction stage should cause some "switch over" to the across-attribute strategy.

\section{The Effect of Knowledge}

Effort itself varies from consumer to consumer and from product to product. Consider two consumers, one naive and one an expert, regarding their knowledge of a pair of products. Each consumer is confronted with concrete representations of the alternatives. To use the within-attribute strategy these representations must be abstracted to a comparable level. The first stage of abstraction is identical for novice and expert, as both consumers must construct a set of applicable attributes. While the expert has more knowledge about the individual alternatives, neither has an advantage with respect to a choice between the alternatives. At the second stage, where attribute values are formed, the expert may have an advantage. 
Let us consider the two cognitive processes identified earlier for obtaining attribute values: recalling values and constructing values. When knowledge of choice alternatives is high, abstract attribute values already stored in memory are simply recalled as needed. The consumer simply "knows" how entertaining a stereo is. When knowledge is low, the effort to measure alternatives on new, more abstract attributes may be very great. Here the consumer consciously constructs abstract attribute values by "mapping" concrete attribute values into the abstract attributes-for example, "how much entertainment value does a TV with a 25 -inch color picture provide?" (Note that this is again quite different from combining values across attributes in the across-attribute strategy.)

If the effort to construct a value is larger than the effort to recall the same value, the effort to abstract and use the within-attribute strategy should decrease with knowledge. Consequently, all else being equal, experts may be more likely than non-experts to use the within-attribute strategy. Knowledge should also, however, affect the effort required to use the acrossattribute strategy. Experts may be more likely to use across-attribute strategies because of affect referral, where consumers easily recall pre-stored overall evaluations or affects for different products or product types (Bettman 1979). As knowledge makes both strategies easier, it may or may not affect their relative use.

\section{Phased Strategies}

The two general strategies, across-attribute and within-attribute, are not mutually exclusive. Consumers may combine the two into a "phased" strategy. One strategy may be used on a subset of relevant attributes in one phase of the decision. The other strategy may then be used on the remaining attributes in a second phase. If the two general strategies do not describe how consumers compare noncomparable alternatives, such phased strategies might be explored.

\section{HYPOTHESES}

Consumers faced with noncomparable alternatives use one of two general strategies: a within-attribute strategy with abstraction or an across-attribute strategy. While the hypothesized use of a within-attribute strategy with abstraction is new, the use of across-attribute strategies is not. Thus a null hypothesis is that, when alternatives are noncomparable, consumers shift entirely to an across-attribute strategy. Accordingly, consumers retain an initially low level of abstraction, combine values across-attributes, and make comparisons on overall evaluations. The level of abstraction of product comparisons shifts, therefore, to an extremely abstract level when alternatives are noncomparable.
Alternatively, consumers may retain a within-attribute strategy and gradually switch to an acrossattribute strategy as comparability decreases. The relative ease of within-attribute comparisons may cause consumers to retain a within-attribute stategy. The added abstraction stage of processing required to use the strategy eventually results in the shift to an acrossattribute strategy.

Retaining a within-attribute strategy while shifting to an across-attribute strategy implies that the more noncomparable the alternatives, the more abstract the resulting product comparisons. Increased use of comparisons on overall evaluations through use of an across-attribute strategy implies increasingly abstract comparisons. Retaining a within-attribute strategy also requires more abstract within-attribute comparisons as alternatives become more noncomparable. Such a gradual shift also implies that while within-attribute comparisons continue to be made, they decrease while the relative amount of across-attribute processing increases. This alternative hypothesis is thus broken into two parts:

H1a: The level of abstraction of product comparisons should increase as alternatives become more noncomparable.

H1b: Consequently, within-attribute comparisons should decrease relative to across-attribute processing as alternatives become more noncomparable.

In other words, hypothesizing a gradual shift to acrossattribute processing implies that a likely consequence would be a shift to higher level comparisons (H1a) and less within-attribute comparison relative to acrossattribute processing ( $\mathrm{H} 1 \mathrm{~b})$.

Knowledge should also affect the effort involved in using the two strategies. Since the relative effect of knowledge on effort is unknown, a second null hypothesis is that knowledge has an equal effect on the effort to use the strategies. Accordingly, knowledge does not affect relative use of the strategies and, consequently, does not affect the level of abstraction of comparisons at a given level of comparability.

\section{METHODOLOGY AND EXPERIMENTS}

Two qualitatively different experiments were used to test the hypotheses: a projective choice task and a controlled choice task. Experiment 1-the projective choice task-presented subjects with scenarios in which a third party is choosing between two specific alternatives. Subjects "think aloud" about how the decisions are made. Experiment 2, the controlled choice task, had subjects make choices involving pairs of hypothetical alternatives. Both verbal protocols and 
eye fixations were collected. Using different methods, individual methods compensate for possible biases inherent in others. Any reasonably valid test of the hypotheses requires more than one method.

The protocols in experiments 1 and 2 are used to determine the level of abstraction of comparisons for products at different levels of comparability. The eye movements in experiment 2 determine the amount of across-attribute processing at different levels of comparability. If consumers "switch over" to the acrossattribute strategy as abstraction effort increases, the eye fixations should reveal an increase in acrossattribute processing from comparable to noncomparable alternatives.

\section{Choice Alternatives and Independent Variables}

Both experiments involve binary choices. The choice alternatives used in the experiments vary with respect to comparability and consumer knowledge, the independent variables of interest. Consumer durables were used because the respondents were likely to vary widely on knowledge about such equipment.

Comparability is operationalized at three levels: comparable, moderately noncomparable, and more noncomparable. At least two noncomparable levels are required to test the hypotheses. If consumers gradually shift to an across-attribute strategy, the level of abstraction of comparisons increases from comparable to moderately noncomparable and from moderately noncomparable to more noncomparable alternatives. If they shift entirely to an across-attribute strategy, comparisons for both moderately noncomparable and more noncomparable alternatives will be very abstract. Comparable choice alternatives are taken from the same product category. As a first approximation, moderately noncomparable and more noncomparable alternatives are operationalized using similarity judgments as a surrogate for comparability. Alternatives from different but similar product categories are classified as moderately noncomparable, while alternatives from different and dissimilar product categories are classified as more noncomparable.

Higher-level classifications were obtained by having a convenience sample of five subjects rate similarities among 12 consumer durables. The sixty-six pairs (with one replication, for a total of 132 judgments) were presented on a CRT with a computer controlled rating scale ranging from 1 (very dissimilar) to 7 (very similar). The general strategy was to identify clusters of similar alternatives. Alternatives within clusters are considered moderately noncomparable, and alternatives across clusters are considered more noncomparable. Visual examination of a three-dimensional representation of the 12 stimuli (stress $=0.041$ ) reveals three clusters-A, B, and C-involving eight of the stimuli. Cluster A contains modes of transportation: bicycles, motorcycles, and automobiles. Cluster B contains home entertainment devices: televisions, stereos, and video cassette recorders. Cluster $\mathrm{C}$ contains domestic appliances: washers and refrigerators. Average intercluster distance is 4.54 times that of average intracluster distance $(1.16$ v. 0.26$)$ with minimum intercluster distance 4.0 times greater than maximum intracluster distance. According to this classification, for example, two televisions are comparable, a television and a stereo are moderately noncomparable, and a television and an automobile are more noncomparable.

Twenty-two choice pairs were used from this classification: eight comparable, seven moderately noncomparable, and seven more noncomparable. Moderately noncomparable alternatives included automobile/bicycle, bicycle/motorcycle, automobile/motorcycle, stereo/television, stereo/video recorder, television/video recorder, and refrigerator/washer. More noncomparable alternatives included bicycle/washer, bicycle/stereo, refrigerator/television, video recorder/motorcycle, washer/stereo, automobile/television, and automobile/refrigerator. One comparable pair served as a warmup.

Data from ten pilot subjects in experiment 1 revealed some problems with this classification. Two choice pairs-automobile/television and automobile/refrigerator-varied more on price than did other noncomparable pairs. Also, the motorcycle/automobile pair seemed more comparable than the classifications indicated while the washer/refrigerator pair seemed less comparable. All four pairs, two each from the noncomparable levels, were not presented to subjects in experiment 2 . In addition, two comparable pairs (motorcycle/motorcycle and television/television) were not included in experiment 2 to equalize the number of stimulus pairs across comparability levels.

Knowledge is controlled indirectly, and varies both across product categories (e.g., refrigerators and video cassette recorders) and across individuals within a product category (e.g., those who own and those who do not own video cassette recorders). Knowledge could be manipulated in a long term, longitudinal study and controlled directly. As a simpler alternative, we measured knowledge for each individual in each product category. Self-ratings were obtained from subjects after they performed the experimental tasks. Measured knowledge relates directly to knowledge of a product's attributes at all levels of abstraction. Knowledge should increase with use, purchase, and thoughtful consideration of the product.

Previous studies have measured knowledge or familiarity differently. For example, Bettman and Park (1980) used a combined measure based on product use, ownership, and information search. As a manipulation check, they used self-rated familiarity using a five point scale from unfamiliar to very familiar. 
EXHIBIT

SAMPLE KNOWLEDGE MEASUREMENT SCALE

$0=$ People who have never used or seen anyone use and are completely ignorant of the product

5 = People who have never used or really considered the product but have seen other people use it and know at least what it may be used for.

$10=$ People who have used the product but have not purchased it for themselves or thoughtfully considered its details and functions.

15 = People who have used the product extensively, purchased it for themselves and thoughtfully considered its details and functions.

$20=$ People who know every aspect of the product and its uses at the level of an engineer or professional in the field.

Johnson and Russo (1984) used a five point scale to have subjects rate previous knowledge for particular products compared to the rest of the population.

For a self-report rating of knowledge involving noncomparable alternatives, certain requirements should be met. First, measured knowledge should be absolute. Since product categories differ in both mean level and range of consumer knowledge, a relative rating is ambiguous. Second, it should cover the entire range of possible knowledge, from that of the ignorant consumer to that of the expert. Third, it should be as specific as possible to avoid self-interpretation of the scale.

The scale used in the two experiments meets these constraints. Absolute levels of knowledge are labeled on a 20-point scale providing a full range of responses. Descriptions of consumers rating $0,5,10,15$, and 20 on the scale are provided next to each scale value (see Exhibit). This scale provides a more comparable measure of knowledge across product categories than a relative scale. The measure is, however, limited. While self-ratings of knowledge are easy to obtain, they may not measure actual knowledge. How much people think they know about products may differ from what they actually know (Park and Lessig 1981).

Knowledge for pairs is obtained by simply averaging across the ratings for alternatives in a pair. Alternatively, usable knowledge may be constrained by the lower of the two ratings. Recall of either attribute values or an overall evaluation may not be useful if it is only possible for one of two alternatives. Knowledge measured as the smaller of the two knowledge ratings for a pair is thus also tested.

\section{EXPERIMENT 1: PROJECTIVE CHOICE TASK}

\section{Method}

In the projective choice task, consumers are presented third-party choice scenarios. Using a thirdparty format prevents consumers from simply recalling the output of a previous decision and refusing to decide due to a very dominant preference relation, prompting a comparison strategy. Twenty-two scenarios were used, one for each choice pair. Each scenario required a consumer to make a decision between the two alternatives. For example, the scenario for bicycle/ stereo was:

Susan received enough money on her birthday to buy herself one nice present, either a new bicycle or a new stereo. Having decided both which bicycle and which stereo she would buy, she must now decide between the two. How do you think Susan will make the decision?

Each scenario ended with a statement asking how the decision is made. The instructions asked for the attributes or criteria considered and how they are used. Subjects were instructed to "think aloud" in response to each scenario.

While a third party format may increase evaluation, there is a danger that the strategies selected may be those the subjects can easily justify for use by others rather than those they themselves would actually use. For example, Tversky (1969) suggests ease of justification as another possible criterion for use of withinattribute strategies.

\section{Subjects and Procedure}

Scenarios were presented individually to eleven subjects, seven female and four male University of Chicago students, who were paid for their participation. After reading the instructions and answering questions regarding procedure, the experimenter read the scenarios out loud, one at a time, while the subject read along. Responses were tape recorded and later transcribed into text, providing a protocol for each subject. After responding, subjects rated product category knowledge and then the likelihood of facing the decisions in the scenarios. Subjects using a third party format were told that they may not bring their own knowledge to bear on the task; they were instructed to answer as though the third party "knows what you know" about the products in question.

\section{Analysis}

To test the hypotheses, protocols were coded for both attributes used and the existence/nonexistence of product comparisons on the attributes. Judges 
coded all mentioned attributes that described the choice alternatives. Only attributes that were mentioned were coded. Mentioned attributes were coded for whether a product comparison occurred on the attribute or whether the attribute was used to simply describe one or both of the alternatives. For example, a statement such as "the motorcycle is not as safe as the automobile" was coded as a relative comparison on the attribute "safety." The instructions allowed limited coding of similar attributes under one label. For example, both "more dangerous" and "safer" were coded as relative comparisons on "safety." (A complete copy of the coding instructions and attribute labels may be obtained from the author.)

The protocols were coded by two independent judges naive to the research hypotheses. Only data for which both judges agreed on both the attribute and its use were used to test the hypotheses. Interjudge reliability was high: they agreed on 717 attributes. Judge 1 coded 67 attributes not coded by judge 2 who, in turn, coded 70 attributes not coded by judge 1 . Taken as the probability that an attribute coded by one judge is coded by the other judge, interjudge reliability was 0.91 .

The classification of attribute use was also checked for reliability (i.e., whether a relative comparison on an attribute occurred or, if not, whether the attribute applied to one or both alternatives). Of the 717 attributes agreed upon, classifications were identical 95 percent of the time. Cohen's measurement of the reliability of the judges' classifications $-K=0.93$-is significantly greater than zero $(p<0.0001)$, where $K$ varies from zero to one and equals zero when classifications are completely independent (Bishop, Fienberg, and Holland 1975, p. 395).

Critical to testing the hypotheses is the abstractnessconcreteness of the nonprice attributes on which comparisons occurred. Since no measure exists, attribute concreteness-abstractness was operationalized by having separate, independent judges rate the attributes elicited by subjects in the experiments. (A total of 249 different attributes was elicited in experiments 1 and 2 ). Judges were instructed to rate each attribute's concreteness-abstractness on an 11-point scale from 0 (very concrete) to 10 (very abstract). Eleven University of Michigan undergraduate marketing students served as judges. Judgments were collected using a paper and pencil format. Three of these judges were dropped due to low average correlations with the other judges $(r=0.48)$. The average interjudge correlation among the eight remaining judges was 0.70 . Abstractness-concreteness was obtained by averaging over the eight judges.

Coded attributes rated for concreteness-abstractness provide a manipulation check of choice pair comparability. Comparability-or attribute overlap-is operationalized as the ratio of attributes common to the alternatives to the average number of distinctive attributes per alternative. Thus when alternatives have more attributes in common with each other than they have distinctive attributes, comparability is greater than one. To avoid the possibility of mistakes affecting the results, only attributes mentioned at least twice in connection with any given alternative were considered.

The results are consistent with the similarity judgments. Most moderately noncomparable pairs have overall comparabilities greater than one, while most more noncomparable pairs have less than one. Only two pairs-video recorder/television and washer/ stereo-have measures inconsistent with this pattern. While the analysis that follows includes these pairs, the sensitivity of the results to their inclusion is also reported.

Given the within-subjects nature of the design, a repeated measures analysis of variance model tests for differences in the dependent variable-the level of abstraction of product comparisons-with respect to the independent variables. The independent variables included for testing the hypotheses are comparability (three levels) and consumer knowledge. Analysis of covariance is performed on knowledge, treated as a continuous variable ranging from 0 to 20. A comparability-by-knowledge interaction term determines whether level of comparison at different levels of comparability depends on knowledge. Also included in the model is a subjects factor (11 levels) and a factor for the individual product pairs (22 levels). Comparability, knowledge, and product pair are all within-subject factors.

\section{Results}

The ANOVA results for experiment 1 are presented in Table 1. Significant main effects exist for both comparability and the individual product pairs. Whether level of comparison increases with comparability or shifts entirely to an abstract level for both moderately and more noncomparable alternatives is critical in testing the hypothesis regarding strategy use. A Newman-Keuls test for differences in means reveals significant differences in level of comparison between comparable and moderately noncomparable and between moderately noncomparable and more noncomparable alternatives (mean level of abstraction of comparisons equals $4.33(n=86), 5.95(n=63)$, and $7.11(n=64)$, respectively, for comparable, moderately noncomparable, and more noncomparable alternatives; differences significant at $p<0.01$ ).

This gradual increase in the abstractness of comparisons supports Hypothesis 1a. Apparently, consumers do not shift completely to an across-attribute strategy. Further qualitative support is provided by the comparisons themselves. The most common comparisons for moderately noncomparable pairs were on 
TABLE 1

REPEATED MEASURES ANALYSIS OF VARIANCE

\begin{tabular}{|c|c|c|c|c|}
\hline Source & df & Mean square & $F$ & $\mathrm{p}$ \\
\hline \multicolumn{5}{|c|}{ Experiment 1: Projective choice task } \\
\hline \multicolumn{5}{|l|}{ Between subjects } \\
\hline Subjects & 10 & 448.999 & 1.58 & .1162 \\
\hline \multicolumn{5}{|l|}{ Within subjects } \\
\hline Comparability & 2 & $14,552.734$ & 51.17 & .0001 \\
\hline Product pair & 20 & 577.434 & 2.03 & .0080 \\
\hline $\begin{array}{l}\text { Knowledge } \\
\text { Comparability }\end{array}$ & 1 & 2.023 & 0.01 & .9329 \\
\hline$\times$ Knowledge & 2 & 14.862 & 0.05 & .9491 \\
\hline \multicolumn{5}{|c|}{ Experiment 2: Controlled choice task } \\
\hline \multicolumn{5}{|l|}{ Between subjects } \\
\hline Subjects & 11 & $1,025.484$ & 4.00 & .0001 \\
\hline \multicolumn{5}{|l|}{ Within subjects } \\
\hline Comparability & 2 & $24,645.289$ & 96.24 & .0001 \\
\hline Product pair & 13 & 350.569 & 1.37 & .1876 \\
\hline Knowledge & 1 & 0.058 & 0.00 & .9880 \\
\hline \multicolumn{5}{|l|}{ Comparability } \\
\hline$\times$ Knowledge & 2 & 80.867 & 0.32 & .7294 \\
\hline
\end{tabular}

"use" (7) and "commuting" (5). The most common comparisons for more noncomparable pairs were on "necessity" (19) and "importance" (4). While the abstractness of comparisons increases, they certainly do not all represent overall evaluations.

Knowledge and a knowledge-by-comparability interaction have no effect on level of comparison, which is consistent with the second null hypothesis. Apparently, consumers use the strategies to approximately the same degree, independent of knowledge. Though not reported, substituting for average knowledge the lower of the two knowledge ratings in a product pair does not affect the results. Neither does excluding from the analysis the two product pairs with problematic comparability measures-washer/stereo and television/video recorder.

While the results have focused on nonprice attributes, recall that price is one concrete attribute on which noncomparable alternatives can be directly compared. To avoid abstracting, consumers may rely more on price comparisons as the comparability of choice alternatives on nonprice attributes decreases. Noncomparable alternatives did result in relatively more price comparisons. The proportion of price comparisons to total (price and nonprice) comparisons in the protocols was 10.4 percent (10 of 96) for comparable alternatives, 21.3 percent (17 of 80 ) for moderately noncomparable alternatives, and 13.5 percent (10 of 74) for more noncomparable alternatives.

This result is confounded, however, by the fact that variance on price-not comparability-may be the major cause of price comparisons. The noncomparable alternatives tended to vary more on price than did the comparable alternatives. For example, protocols involving noncomparable alternatives for which price differences are comparatively large (automobile/motorcycle, automobile/bicycle, automobile/television, automobile/refrigerator, and motorcycle/bicycle) contained 22.5 percent price comparisons (16 of 71) compared to 13.3 percent (11 of 83 ) for the remaining noncomparable alternatives. This explains why the moderately noncomparable alternatives resulted in more price comparisons than did the more noncomparable alternatives: twelve of the seventeen price comparisons at this level were on the high price difference pairs. Further research on the effects of comparability versus price variance on the use of price comparisons is needed.

In summary, a projective test reveals significant increases in the abstractness of product comparisons for increasingly noncomparable alternatives. The data supports the alternative hypothesis that subjects retain a within-attribute strategy and gradually shift to an across-attribute strategy. The results can not reject the null hypothesis that product knowledge has no effect on level of comparison. Consumers apparently use the two strategies to the same degree across knowledge levels.

\section{EXPERIMENT 2: CONTROLLED CHOICE TASK}

\section{Method}

The controlled choice task presents consumers with choices involving pairs of hypothetical alternatives. The sixteen choice pairs outlined earlier served as stimuli. Alternatives were described on three concrete, nonprice attributes and also on price. Nonprice attributes included the three most frequently mentioned attributes for an alternative in the range 0.0 to 1.0 of concreteness-abstractness (using data obtained from the ten pilot subjects in experiment 1 rated on a 0.0 to 10.0 scale). Attribute values were taken from the range of common values for the respective products in Consumer Reports. Prices were assigned to be consistent with the attributes of the products (the higher the attribute values the higher the price). Extreme attribute values were avoided. Comparable choice pairs were constructed so as to include no dominated alternatives (i.e., where one alternative was superior on all attributes) to facilitate evaluation.

Dependent measures included verbal protocols and eye fixations. If Hypothesis $1 \mathrm{~b}$ is true, the eye fixations should reveal an increase in the proportion of acrossto within-attribute processing as comparability decreases. While the eye fixations may reveal this shift, keep in mind that abstraction in the within-attribute strategy may itself resemble a holistic or across-attri- 
bute process. For example, mapping concrete attribute values into abstract attribute values may occur across attributes. An increase in across-attribute eye fixations with a decrease in comparability may simply indicate the abstraction process required to use the withinattribute strategy.

Two manipulations facilitated evaluation. First, the third-person scenarios in experiment 1 were given in first-person format to subjects in experiment 2. By providing a scenario in which the subject faces the choice at hand, the likelihood of overt evaluation increases. Second, subjects were asked both to choose and to indicate strength of preference on a scale of 1 (slight) to 4 (strong).

\section{Subjects and Procedure}

Subjects included seven female and five male University of Chicago students paid for their participation. The experiment was conducted individually on each subject. The experimenter read through the instructions with each subject, answered any questions, and then read the choice scenarios one at a time. After each scenario was read, a corresponding choice pair was presented on a television monitor in front of the subject. One comparable choice pair served as practice; the remaining fifteen pairs were then presented in random order. Choice pair order was reversed for half the subjects, as was left/right position of the alternatives.

The procedure allowed simultaneous collection of eye fixations and verbal protocols. The "think aloud" protocols were tape recorded and transcribed into text. Eye fixations were monitored using an Applied Science Laboratory 1996 Eye View Monitoring System in the Consumer Behavior Laboratory at the University of Chicago. Fixations were recorded on a video cassette recorder. To facilitate eye fixation collection, subjects were placed in a chair with a headrest which restricts movement without discomfort. Subjects were free to move their heads (the constraint is subject-controlled, not experimenter-controlled) whenever they felt the need to.

\section{Analysis}

As in experiment 1, protocols were coded for both attributes used and existence/nonexistence of product comparisons. Coding instructions from experiment 1 were changed to the first person and used here. The two judges from experiment 1 coded the protocols. Both judges were naive regarding the research hypotheses. As in experiment 1, only commonly coded data was used to test the hypotheses. Interjudge reliability was again high. Judges agreed on 746 attributes, while judge 1 coded thirty-two attributes not coded by judge 2 who, in turn, coded forty-five attributes not coded by judge 1 . Interjudge reliability (the probability that an attribute coded by one judge is coded by the second judge) was 0.95 . As for attribute use, judges agreed on 96 percent of the classifications, and Cohen's measure of classification agreement, $K$ $=0.96$, is again significantly greater than zero ( $p$ $<0.0001$ ).

Video recorded eye fixations were also coded. The two judges who coded the protocols also coded the fixations. One judge coded all eleven possible subjects (one subject's data was lost because of a bad tape). A second judge coded a subset of three subjects as a reliability check. Judges were instructed to code all fixations lasting a minimum of 250 milliseconds-the minimum time necessary to recognize information (Massaro 1975). The existence of fixation patterns indicating within- or across-attribute processing - specifically patterns of three or more consecutive fixations (see Russo and Rosen 1975)-is important. Given alternatives $\mathrm{A}$ and $\mathrm{B}$ with attributes $\mathrm{A} 1, \mathrm{~A} 2, \mathrm{~A} 3, \mathrm{~A} 4$, and $\mathrm{B} 1, \mathrm{~B} 2, \mathrm{~B} 3, \mathrm{~B} 4$, respectively, triplets of the form $\mathrm{Ai}-\mathrm{Aj}-\mathrm{Ai}$ or $\mathrm{Ai}-\mathrm{Aj}-\mathrm{Ak}$ indicate across-attribute processing, while triplets of the form Ai-Bi-Ai indicate within-attribute processing. Coder reliability-the probability a sequence coded by one judge is also coded by the second judge-was 0.81 . Reliability was identical for both within-attribute sequences (Ai-Bi$\mathrm{Ai}$ ) and across-attribute sequences ( $\mathrm{Ai}-\mathrm{Aj}-\mathrm{Ak}$ or $\mathrm{Ai}-$ Aj-Ai).

Again, the within-subjects nature of the design dictates a repeated measures analysis of variance. The ANOVA model tested in experiment 1 is also tested in experiment 2 . The model includes subjects (twelve levels), comparability (three levels), knowledge (continuous variable), individual product pairs (fifteen levels), and a comparability by knowledge interaction. The eye fixations test for the proportion of acrossattribute to total meaningful (across plus within) fixation sequences at different levels of comparability. Bartholomew's test for ordered proportions (see Fleiss 1973, pp. 100-102) tests the hypothesis that the proportion of across-attribute to within-attribute sequences increases from comparable to noncomparable level alternatives. This analysis is also performed within subjects.

\section{Results}

The ANOVA results for experiment 2 are presented in Table 1. Consistent with experiment 1 , a significant main effect exists for comparability with no effect for knowledge or a knowledge by comparability interaction. The subject factor is significant while the product pair factor is not. Again, of some importance to the comparability main effect is whether level of comparison increases the more noncomparable the alternatives. Also consistent with experiment 1 , a Newman- 
Keuls comparison of the means reveals a significant increase in the abstractness of comparisons from comparable to moderately noncomparable and from moderately noncomparable to more noncomparable alternatives (mean level of comparison equals 2.25 ( $n$ $=69), 5.88(n=36)$, and $7.25(n=24)$, respectively, for comparable, moderately noncomparable, and more noncomparable alternatives; differences are significant at $p<0.01$ ).

That consumers retain a within-attribute strategy and gradually shift to an across-attribute strategy is supported. Retainment of within-attribute comparisons is again supported by the comparisons themselves, which did not all represent overall evaluations. The most common comparison for moderately noncomparable pairs was on "mobility" (6). The most common comparisons for more noncomparable pairs were on "necessity" (5), "use" (5), and "importance" (3). As in experiment 1 , neither substituting minimum withinpair knowledge for average knowledge nor dropping washer/stereo and television/video recorder from the analysis affects the pattern of results.

The eye fixations support the switchover from within- to across-attribute processing as comparability decreases. These data are presented in Table 2. Withinattribute comparisons on price are excluded. The proportion of across-attribute to total meaningful sequences is given for each subject at each level of comparability. The proportion increases significantly from comparable to noncomparable alternatives for eight of the eleven subjects $(p<0.05)$. These data support Hypothesis $1 \mathrm{~b}$ and a shift to an across-attribute strategy.

Interestingly, five of the 11 subjects in Table 2 used mostly across-attribute processing on nonprice attributes even when alternatives were comparable. As discussed earlier, the ease of within-attribute comparisons does not always imply preference for a withinattribute strategy. While the eye fixations reveal increased across-attribute processing for noncomparable alternatives, recall that abstraction in the withinattribute strategy may itself resemble a holistic or across-attribute process. Consistency of the results with the hypothesized tradeoff is nonetheless promising and consistent with the analysis of variance results.

The combined data from experiments 1 and 2 test for the average number of comparisons made at each level of comparability. Both the average number of attributes mentioned and the number of comparisons made per choice pair decreased with increased noncomparability of the alternatives $(3.9,3.2$, and 2.9 attributes mentioned and $1.3,1.1$, and 0.8 comparisons made, respectively, for comparable, moderately noncomparable, and more noncomparable alternatives). Both averages differ significantly $(F=12.15, \quad p$ $<0.001$ for attributes mentioned; $F=7.70, p<0.001$ for comparisons made). The decrease in comparisons
TABLE 2

PROPORTION OF ACROSS-ATTRIBUTE TO TOTAL EYE FIXATION SEQUENCES IN EXPERIMENT 2

\begin{tabular}{clccc}
\hline \hline & \multicolumn{5}{c}{ Alternatives } \\
\cline { 2 - 4 } Subject & Comparable & $\begin{array}{c}\text { Moderately } \\
\text { noncomparable }\end{array}$ & $\begin{array}{c}\text { More } \\
\text { noncomparable }\end{array}$ & $\begin{array}{c}\text { Chi- } \\
\text { square }\end{array}$ \\
\hline 1 & $.650(20)^{b}$ & $.750(16)$ & $.905(21)$ & 3.846 \\
2 & $.320(25)$ & $.850(20)$ & $.816(38)$ & 20.587 \\
3 & $.176(17)$ & $.500(12)$ & $.737(19)$ & 11.324 \\
4 & $.286(7)$ & $.889(9)$ & $.800(15)$ & 7.875 \\
5 & $.714(28)$ & $.778(9)$ & $.636(11)$ & $0.000^{c}$ \\
6 & $.500(10)$ & $1.000(7)$ & $.625(8)$ & $2.482^{c}$ \\
7 & $.526(19)$ & $.667(9)$ & $.676(37)$ & $1.272^{c}$ \\
8 & $.625(8)$ & $1.000(8)$ & $.923(13)$ & 6.580 \\
10 & $.421(19)$ & $.786(14)$ & $.826(23)$ & 8.804 \\
11 & $.385(13)$ & $1.000(2)$ & $.857(7)$ & 5.639 \\
12 & $.143(7)$ & $1.000(2)$ & $.833(6)$ & 8.037 \\
\hline
\end{tabular}

- Chi-square obtained using Bartholomew's test for order.

${ }^{b}$ Total number of sequences given in parentheses.

${ }^{\circ}$ Not significant, alpha $=0.05$.

again supports Hypothesis $1 \mathrm{~b}$-that within-attribute comparisons decrease for more noncomparable alternatives. Combined with the ANOVA and eye fixation data, strong support is provided for subjects retaining a within-attribute strategy and gradually shifting to an across-attribute strategy as comparability decreases.

Finally, the protocols were again checked for the use of price comparisons at different levels of comparability. Experiment 2 produced more price comparisons, on average, than did experiment 1 (37 of 250 , or 14.8 percent, for experiment 1 compared to 82 of 211 , or 38.9 percent for experiment 2). This is not surprising, because price was provided for subjects in experiment 2 while no attribute information was given for subjects in experiment 1 . The proportion of price comparisons to total comparisons increased slightly as comparability decreased (42 of 111 , or 37.8 percent, for comparable alternatives; 23 of 59 , or 39 percent, for moderately noncomparable alternatives; and 17 of 41 , or 41.5 percent, for more noncomparable alternatives). Again, variance on price may be driving this result.

To summarize, a controlled choice task reveals significant increases in the abstractness of product comparisons for increasingly noncomparable alternatives. The proportion of across-attribute to total meaningful eye fixation sequences also increases with noncomparability for most subjects. Product knowledge had no effect on level of comparison. These results are consistent with those from experiment 1 and support the hypothesis that consumers retain a within-attribute strategy and eventually shift to an across-attribute strategy as comparability decreases. Both experiments 1 and 2 fail to reject the hypothesis that knowledge has no effect on level of comparison. 


\section{DISCUSSION}

This research takes a necessary first step by determining how people compare noncomparable alternatives. The subjects in the experiments retainedthough made decreasing use of $-\mathrm{a}$ within-attribute strategy as the comparability of alternatives decreased. People often prefer within-attribute processing and may choose a problem representation that allows it.

Additional support for consumers retaining a withinattribute strategy for noncomparable alternatives is provided by a third experiment reported by Johnson (1983). Using a questionnaire format, subjects were asked directly how they evaluate and compare comparable and noncomparable alternatives. The scenarios used in experiment 2 of this study were presented to subjects along with descriptions of the products. The scenarios were followed by hypothetical protocols of individual choices among the alternatives-each protocol corresponding to a particular strategy. Subjects then chose the protocol (strategy) that best described how they would evaluate the alternatives. Three strategy protocols were available: an across-attribute strategy, a within-attribute strategy with abstraction, and a phased strategy combining across- and within-attribute processing. Consumers chose the within-attribute strategy protocol in 63 percent of the cases involving noncomparable alternatives.

Why people would retain a within-attribute strategy rather than completely switch to an across-attribute strategy is not clear. Several explanations are offered. One is that the within-attribute strategy is more consistent with minimization of effort. Since withinattribute comparisons are relatively easy, the withinattribute strategy may require less effort. This particular advantage outweighs any added effort required to form abstract product representations or perform other operations.

A second explanation is that error is minimized by using the within-attribute strategy. Consider the precision of values on attributes varying in concretenessabstractness. Perhaps it is true that the more abstract the attribute, the less precise the values of the attribute, and the greater the potential for error when comparing alternatives. Consider, for example, a car getting 25.5 miles per gallon of gasoline versus a car that is "economical." Overall evaluations produced by an across-attribute strategy are quite abstract, while within-attribute comparisons are, on average, less abstract. If within-attribute comparisons are more precise than comparisons on overall evaluations, consumers may use the within-attribute strategy because it leads to fewer errors rather than less effort, or a combination of the two. While plausible, this explanation is highly speculative and requires further testing.

A third explanation is that one can not assume that consumers can compare overall evaluations across product categories. Overall evaluations may only have meaning relative to a specific product category or group: they may not lend themselves to comparisons across categories. For example, determining that Budweiser is a good beer and Coke is a very good soft drink may not imply that Coke is better than Budweiser. While concepts like utility serve their purpose, they may have little or no intuitive appeal to consumers. The most abstract, meaningful comparisons across categories may be on attributes such as necessity and luxury.

Two explanations call into question whether error/ effort minimization guides strategy selection. Withinattribute strategies-such as additive difference-may be chosen because they are easier for subjects to justify using (Tversky 1969). Finally, while the across-attribute strategy may be possible and may minimize error and effort, it simply may not be obvious to many consumers that such a strategy exists or can be used. Consumers may see a within-attribute strategy as the only immediate way of structuring the problem. Seeing that it will work, they use it. They do not consider that an across-attribute strategy is also feasible and, perhaps, better.

Why do consumers appear to use a within-attribute strategy to the same degree across knowledge levels? According to the null hypothesis, knowledge has a roughly equal effect on the effort to use the strategies. Even when the minimum knowledge level for each pair of alternatives was used in the analysis, the null hypothesis was supported. That knowledge produced no effects on level of comparison is nonetheless curious. If knowledge is constrained by the minimum knowledge level among the alternatives, a floor effect is possible with all respondents reporting low knowledge. It may be rare for subjects to have a high level of knowledge-or be an "expert"-on alternatives from two or more different product categories. However, even when the lower of the two knowledge ratings for a pair of alternatives was used, 38 and 8 percent, respectively, of the noncomparable pairs in the two experiments fell in the upper half and upper quarter of the knowledge scale (compared to 63 and 21 percent, respectively, assuming average knowledge). Finally, as cautioned earlier, self-reported measures of knowledge may not reflect differences in actual product knowledge.

\section{SUMMARY}

The results support Hypotheses $1 \mathrm{a}$ and $1 \mathrm{~b}$. As alternatives become noncomparable, consumers retain a within-attribute strategy by abstracting product representations to a level where comparisons are possible, while also shifting to an across-attribute strategy. The results do not reject the hypothesis that knowledge has no effect on the relative use of the two strategies. 
These results are consistent across independent and qualitatively different experiments.

While research on consumer choice strategies has been limited to comparable alternatives, consumers choose among both comparable and noncomparable alternatives. It is important to study the strategies used on noncomparable alternatives, which involve abstract product attributes, in order to expand the scope of choice strategy research. This research, and information processing research in general, has been criticized for its inability to address consumption decisions involving abstract or "experiential" attributes (Holbrook and Hirschman 1982). As the research reported here demonstrates, choice strategy research may be limited more by the problem areas that have been studied than by the nature of the underlying, information processing paradigm.

Choice strategy research is also generally concerned with describing the strategies people use. Future research should explore the power of strategy error and effort to both explain and predict choice strategy selection. As one explanation of the results indicates, further specification of error may be crucial in explaining and predicting strategy selection.

Consider two examples of why decision error is far from simple. First, decisions are often interdependent. For example, in a constantly changing environment, choosing randomly with minimum effort tends to reduce future errors. Random choice allows consumers to obtain important information for subsequent decisions (March 1978). Second, minimization of error and effort are not necessarily incompatible. In a family decision-making context, maintenance of the family may be as important as finding a better alternative when making a decision (Davis 1976). If finding a better alternative only leads to conflict among family members, minimizing effort may be consistent with minimizing error. Future research should continue to explore error to discover what it is composed of, whether it can be measured, when error and effort trade off, and when they are compatible.

Further specification of the strategies and the error and effort tradeoffs involved in the strategy selection process is an important next step. A model of the error and effort involved in each strategy is currently being tested and estimated. The results yield predictions about strategy selection in various contexts, including binary and multialternative choices involving both comparable and noncomparable alternatives. Testing these predictions in both laboratory and more natural choice environments is a necessary progression.

Finally, while posited here in the context of consumer choice, comparability is important to a wide variety of the decisions we face involving other than consumer goods and groups and individuals. It is hoped that continued empirical testing of the strategies outlined here and their predictions will improve our knowledge of consumer behavior and of behavioral decision making in general.

\section{[Received February 1984. Revised September 1984.]}

\section{REFERENCES}

Berning, Carol A., and Jacob Jacoby (1974), "Patterns of Information Acquisition in New Product Purchases," Journal of Consumer Research, 1 (September), 18-22.

Bettman, James R. (1974), "Decision-Net Models of Buyer Information Processing and Choice: Findings, Problems, and Prospects," in Buyer/Consumer Information Processing, eds. G. D. Hughes and M. L. Ray, Chapel Hill, NC: University of North Carolina Press, 59-74.

- (1979), An Information Processing Theory of Consumer Choice, Reading, MA: Addison-Wesley.

- and Jacob Jacoby (1976), "Patterns of Processing in Consumer Information Acquisition," in Advances in Consumer Research, Vol. 3, ed. Beverlee B. Anderson, Ann Arbor, MI: Association for Consumer Research, 315-320.

_ and Pradeep Kakkar (1977), "Effects of Information Presentation Format on Consumer Information Acquisition Strategies," Journal of Consumer Research, 3 (March), 233-240.

and C. Whan Park (1980), "Effects of Prior Knowledge and Experience and Phase of the Choice Process on Consumer Decision Processes," Journal of Consumer Research, 7 (December), 234-248.

Bishop, Yvonne M., Stephen E. Fienberg, and Paul W. Holland (1975), Discrete Multivariate Analysis: Theory and Practice, Cambridge, MA: The MIT Press.

Davis, Harry L. (1976), "Decision Making Within the Household," Journal of Consumer Research, 2 (March), 241-260.

Einhorn, Hillel J. (1970), "The Use of Nonlinear, Noncompensatory Models in Decision Making," Psychological Bulletin, 73 (3), 221-230.

Fleiss, Joseph L. (1973), Statistical Methods for Rates and Proportions, New York: John Wiley.

Hogarth, Robin M. (1975), "Decision Time as a Function of Task Complexity," in Utility, Probability, and Human Decision Making, eds. Wendt/Vlek, Reidel.

Holbrook, Morris B. and Elizabeth C. Hirschman (1982), "The Experiential Aspects of Consumption: Consumer Fantasies, Feelings, and Fun," Journal of Consumer Research, 9 (September), 132-140.

Howard, John (1977), Consumer Behavior: Application of Theory, New York: McGraw-Hill.

Jacoby, Jacob, Robert Chestnut, Karl Weigl, and William Fisher (1976), "Pre-purchase Information Acquisition: Description of a Process Methodology, Research Paradigm, and Pilot Investigation," in Advances in Consumer Research, Vol. 3, ed. Beverlee B. Anderson, Ann Arbor, MI: Association for Consumer Research, 306-314.

_- George Szybillo, and Jacqueline Busato-Schach (1977), "Information Acquisition Behavior in Brand Choice Situations," Journal of Consumer Research, 3 (March), 209-216.

Johnson, Eric J., and John W. Payne (1984), "Effort and Accuracy in Choice," Managerial Science, in press. 
and J. Edward Russo (1984), "Product Familiarity and Learning New Information," Journal of Consumer Research, 11 (June), 542-550.

Johnson, Michael D. (1980), "An Information Processing Analysis of Product Labels," in Advances in Consumer Research, Vol. 7, ed. Jerry C. Olson, Ann Arbor, MI: Association for Consumer Research, 724-728.

(1983), "Decision Processing and Product Comparability: a Theory of Strategy Selection," unpublished Doctoral Dissertation, The University of Chicago.

- (1984), "Modeling Choice Strategies for Noncomparable Alternatives," working paper, The University of Michigan, Graduate School of Business Administration.

Klein, Noreen M. (1983), "Utility and Decision Strategies: A Second Look at the Rational Decision Maker," Organizational Behavior and Human Performance, 31 (February), 1-25.

Kotler, Philip (1984), Marketing Management: Analysis, Planning and Control, 5th ed., Englewood Cliffs, NJ: Prentice-Hall.

Lussier, Denis and Richard Olshavsky (1979), "Task Complexity and Contingent Processing in Brand Choice," Journal of Consumer Research, 6 (September), 154165.

March, James G. (1978), “Bounded Rationality, Ambiguity, and the Engineering of Choice," Bell Journal of Economics, 9 (2), 587-608.

Massaro, Dominic W. (1975), Experimental Psychology and Information Processing, Chicago, Rand McNally.

Paivio, Allan (1971), Imagery and Verbal Processes, New York: Holt, Rinehart and Winston.

Park, C. Whan and V. Parker Lessig (1981), "Familiarity and Its Impact on Consumer Decision Biases and Heuristics," Journal of Consumer Research, 8 (September), 223-230.

Payne, John W. (1976), "Task Complexity and Contingent Processing in Decision Making: An Information Search and Protocol Analysis," Organizational Behavior and Human Performance, 16 (August), 366-387.
Russo, J. Edward (1977), "The Value of Unit Price Information," Journal of Marketing Research, 14 (May), 193-201.

(1981), "The Decision to Use Product Information at the Point of Purchase," in Theory in Retailing: Traditional and Nontraditional Sources, eds. Ronald W. Stampfl and Elizabeth C. Hirschman, Chicago: American Marketing Association.

- and Barbara Dosher (1983), "Strategies for Multiattribute Binary Choice," Journal of Experimental Psychology: Learning, Memory and Cognition, 9 (4), 676696.

and Larry Rosen (1975), “An Eye Fixation Analysis of Multi-Alternative Choice," Memory and Cognition, 3 (3), 267-276.

Sheluga, David A., James Jaccard, and Jacob Jacoby (1979), "Preference, Search, and Choice: An Integrative Approach," Journal of Consumer Research, 6 (September), $166-176$.

Shugan, Steven (1980), "The Cost of Thinking," Journal of Consumer Research, 7 (September), 99-111.

Smead, Raymond J., James B. Wilcox, and Robert E. Wilkes (1981), "How Valid are Product Descriptions and Protocols in Choice Experiments," Journal of Consumer Research, 8 (June), 37-42.

Tversky, Amos (1969), "Intransitivity of Preferences," Psychological Review, 76 (1), 31-48.

(1972), "Elimination by Aspects: A Theory of Choice," Psychological Review, 79 (4), 281-299. - (1977), "Features of Similarity," Psychological Review, 84 (4), 327-352.

van Raaij, W. Fred (1977), “Consumer Information Processing for Different Information Structures and Formats," in Advances in Consumer Research, Vo. 4, ed. William D. Perreault, Jr., Atlanta: Association for Consumer Research, 176-184.

Wright, Peter (1975), "Consumer Choice Strategies: Simplifying vs. Optimizing," Journal of Marketing Research, 12 (February), 60-67. 